\title{
SPECTRAL INCLUSIONS AND STABILITY RESULTS FOR STRONGLY CONTINUOUS SEMIGROUPS
}

\section{ABDELKADER ELKOUTRI and MOHAMED AZIZ TAOUDI}

\author{
Received 12 February 2002
}

\begin{abstract}
We prove some spectral inclusions for strongly continuous semigroups. Some stability results are also established.
\end{abstract}

2000 Mathematics Subject Classification: 47D03, 47A10.

1. Introduction and preliminaries. Let $X$ be a complex Banach space and let $A$ be a closed linear operator with domain $D(A)$, kernel $N(A)$, and range $R(A)$ in $X$. We will say that $A$ is semiregular if $R(A)$ is closed and $N(A) \subseteq R^{\infty}(A)$, where $R^{\infty}(A):=\bigcap_{n=1}^{\infty} R\left(A^{n}\right)$.

Denote the regular spectrum by

$$
\sigma_{\gamma}(A):=\{\lambda \in \mathbb{C}, \lambda-A \text { is not semiregular }\}
$$

The set $\sigma_{\gamma}(A)$ was studied (under various names) by several authors, see for instance $[11,12,13,14]$ and the references therein.

An operator $A$ is said to be essentially semiregular if $R(A)$ is closed and there exists a finite-dimensional subspace $G \subseteq X$ such that $N(A) \subseteq R^{\infty}(A)+G$. Define further the essential regular spectrum of $A$ by

$$
\sigma_{y e}(A):=\{\lambda \in \mathbb{C}, \lambda-A \text { is not essentially semiregular }\} .
$$

This concept was introduced and studied for bounded operators in [13, 17].

We say that $A$ is upper semi-Fredholm if $R(A)$ is closed and $\operatorname{dim} N(A)<\infty$. The left essential spectrum is given by

$$
\sigma_{\pi}(A):=\{\lambda \in \mathbb{C}, \lambda-A \text { is not upper semi-Fredholm }\} .
$$

Let $X^{\star}$ denote the dual space of $X$ and $A^{\star}$ the adjoint operator of $A$. Define the reduced minimum modulus $\gamma(A)$ by setting

$$
\gamma(A):=\inf \left\{\frac{\|A u\|}{d(u, N(A))}, u \in D(A) \backslash N(A)\right\} .
$$


It is well known (see [9]) that $\gamma(A)=\gamma\left(A^{\star}\right)$ and $\gamma(A)>0$ if and only if $R(A)$ is closed. Let $H_{0}(A)$ denote the quasinilpotent part of $A$ given by

$$
H_{0}(A):=\left\{x \in \bigcap_{n \geq 0} D\left(A^{n}\right): \lim _{n \rightarrow \infty}\left\|A^{n} x\right\|^{1 / n}=0\right\}
$$

Let $\mathscr{T}=(T(t))_{t \geq 0}$ be a strongly continuous semigroup with generator $A$ on $X$. We will denote the type (growth bound) of $\mathscr{T}$ by $\omega_{0}$ :

$$
\begin{aligned}
\omega_{0} & :=\lim _{t \rightarrow \infty} \frac{\ln \|T(t)\|}{t} \\
& =\inf \left\{\omega \in \mathbb{R}: \text { there exists } M \text { such that }\|T(t)\| \leq M e^{\omega t}, t \geq 0\right\} .
\end{aligned}
$$

Following [6], the semigroup $\mathscr{T}$ is called bounded if there exists $M \geq 1$ such that $\|T(t)\| \leq M$ for all $t \geq 0$. Basic materials on semigroups may be found in $[4,6,15]$.

In [5], we have studied the regular spectrum for strongly continuous semigroups. As a continuation of [5], the present paper deals with the essentially regular spectrum. Moreover, we establish some stability results for strongly continuous semigroups.

The present paper is organized as follows. In Section 2, we first prove that the spectral inclusion for semigroups remains true for the regular spectrum, the left essential spectrum, and the essentially regular spectrum (Theorem 2.1). Secondly, we give necessary and sufficient conditions for the generator of a strongly continuous semigroup to be semiregular (Theorem 2.3) and essentially semiregular (Theorem 2.5).

In Section 3, we derive some stability results for strongly continuous semigroups. Among other results, we give necessary and sufficient conditions for the generator of a bounded strongly continuous semigroup to have no pure imaginary point in its spectrum (Theorem 3.3). This, in particular, provides us with a spectral characterization of the strong stability of the ultrapower extension of a given semigroup. Finally, we discuss the strong stability of a strongly continuous semigroup via the behavior of the resolvent of its generator, on the imaginary axis.

Throughout this paper, we let $\sigma(A), \rho(A), \sigma_{\mathrm{p}}(A), \sigma_{\mathrm{ap}}(A)$, and $\sigma_{\mathrm{su}}(A)$ denote, respectively, the spectrum, the resolvent set, the point spectrum, the approximative spectrum, and the surjective spectrum of an operator $A$. For $\lambda \in \rho(A)$, $R(\lambda, A)$ denotes the resolvent operator $(\lambda-A)^{-1} \in \mathscr{B}(X)$ of $A$, where $\mathscr{B}(X)$ stands for the algebra of bounded linear operators on $X$.

For later use, we introduce the following operator acting on $X$ and depending on the parameters $\lambda \in \mathbb{C}$ and $t \geq 0$ :

$$
I(\lambda, t) x:=\int_{0}^{t} e^{\lambda(t-s)} T(s) x d s, \quad x \in X
$$


It is well known (see [15]) that $I(\lambda, t)$ is a bounded linear operator on $X$ and we have

$$
\begin{aligned}
e^{\lambda t} x-T(t) x & =(\lambda-A) I(\lambda, t)(x) & & (x \in X) \\
& =I(\lambda, t)(\lambda-A)(x) & & (x \in D(A)) .
\end{aligned}
$$

We conclude this section by the following result which we need in the sequel.

LEMMA 1.1 [10]. If $A \in \mathscr{B}(X)$ is essentially semiregular, then $R^{\infty}(A)$ is closed and the operator $\hat{A}: X / R^{\infty}(A) \rightarrow X / R^{\infty}(A)$ induced by $A$ is upper semiFredholm.

2. Spectral inclusions. In this section, we study the regular spectrum and the essentially regular spectrum of the generator of a strongly continuous semigroup. We begin with the following spectral inclusions.

THEOREM 2.1. For the generator A of a strongly continuous semigroup $(T(t))_{t \geq 0}$, there exist the spectral inclusions

$$
e^{t v(A)} \subseteq v(T(t)) \backslash\{0\}, \quad \forall t \geq 0,
$$

where $v \in\left\{\sigma_{\gamma}, \sigma_{\pi}, \sigma_{\gamma e}\right\}$.

To prove this result, we need the following lemma.

LEMMA 2.2. Let $A$ be the generator of a strongly continuous semigroup $(T(t))_{t \geq 0}$. Then, for all $\lambda \in \mathbb{C}, t \geq 0$, and $n \in \mathbb{N}$,

(i)

$$
\begin{aligned}
\left(e^{\lambda t}-T(t)\right)^{n}(x) & =(\lambda-A)^{n} I(\lambda, t)^{n}(x) & & (x \in X) \\
& =I(\lambda, t)^{n}(\lambda-A)^{n}(x) & & \left(x \in D\left(A^{n}\right)\right) ;
\end{aligned}
$$

(ii) $R^{\infty}\left(e^{\lambda t}-T(t)\right) \subseteq R^{\infty}(\lambda-A)$;

(iii) $N\left((\lambda-A)^{n}\right) \subseteq N\left(\left(e^{\lambda t}-T(t)\right)^{n}\right)$;

(iv) $H_{0}(\lambda-A) \subseteq H_{0}\left(e^{\lambda t}-T(t)\right)$.

Proof of LEMMA 2.2. As mentioned before, $I(\lambda, t)$ is a bounded linear operator on $X$ and we have

$$
\begin{aligned}
e^{\lambda t} x-T(t) x & =(\lambda-A) I(\lambda, t)(x) & & (x \in X) \\
& =I(\lambda, t)(\lambda-A)(x) & & (x \in D(A)) .
\end{aligned}
$$

Proceeding by induction, we get the desired result. The assertions (ii), (iii), and (iv) follow easily from (i).

Proof OF THEOREM 2.1

The Regular SPECTRUM. See [5]. 
THE LEFT ESSENTIAL SPECTRUM. Let $t_{0}>0$ be fixed and suppose that $e^{\lambda t_{0}} \notin$ $\sigma_{\pi}\left(T\left(t_{0}\right)\right)$ for some $\lambda \in \mathbb{C}$. We show that $\lambda \notin \sigma_{\pi}(A)$. Using Lemma 2.2(iii), together with $\operatorname{dim} N\left(e^{\lambda t_{0}}-T\left(t_{0}\right)\right)<\infty$, we infer that $N(\lambda-A)$ is finite dimensional. Now, we prove that $R(\lambda-A)$ is closed. Since $N\left(e^{\lambda t_{0}}-T\left(t_{0}\right)\right)$ is finite dimensional, there exists a closed subspace $Y$ of $X$ such that $N\left(e^{\lambda t_{0}}-T\left(t_{0}\right)\right) \oplus Y=X$. But, $(\lambda-A)\left(N\left(e^{\lambda t_{0}}-T\left(t_{0}\right)\right) \cap D(A)\right)$ is finite dimensional and therefore closed. Then, we need only to show that $(\lambda-A)(Y \cap D(A))$ is closed. From the closedgraph theorem and the closedness of $R\left(e^{\lambda t_{0}}-T\left(t_{0}\right)\right)$, it follows that there is a constant $C>0$ such that

$$
\left\|e^{\lambda t_{0}} x-T\left(t_{0}\right) x\right\| \geq C\|x\|, \quad \forall x \in Y .
$$

From Lemma 2.2(i), we obtain that, for every $x \in D(A)$,

$$
\left\|e^{\lambda t_{0}} x-T\left(t_{0}\right) x\right\| \leq M\|\lambda x-A x\|
$$

for some positive constant $M$. The combination of inequalities (2.4) and (2.5) gives us

$$
\|\lambda x-A x\| \geq \frac{C}{M}\|x\|, \quad x \in Y \cap D(A) .
$$

From the fact that $\lambda-A$ is closed, the result follows.

THE ESSENTIAL REgUlAR SPECTRUM. Let $t_{0}>0$ be fixed and suppose that $e^{\lambda t_{0}}-T\left(t_{0}\right)$ is essentially semiregular for some $\lambda \in \mathbb{C} \backslash\{0\}$. We show that $\lambda-A$ is essentially semiregular. To this end, consider the closed $(T(t))_{t \geq 0}$-invariant subspace $M:=R^{\infty}\left(e^{\lambda t_{0}}-T\left(t_{0}\right)\right)$ of $X$ and the quotient semigroup $(\hat{T}(t))_{t \geq 0}$ defined on $X / M$ by

$$
\widehat{T}(t) \hat{x}:=\widehat{T(t) x}, \quad \text { for } \hat{x} \in X / M,
$$

with generator $\hat{A}$ defined by

$$
D(\widehat{A}):=\{\hat{x}, x \in D(A)\}, \quad \hat{A} \hat{x}:=\widehat{A x}, \quad \forall \hat{x} \in D(\widehat{A}) .
$$

From Lemma 1.1, it follows that the operator $e^{\lambda t_{0}}-\widehat{T}\left(t_{0}\right)$ is upper semiFredholm. Thus, $e^{\lambda t_{0}} \notin \sigma_{\pi}\left(\hat{T}\left(t_{0}\right)\right)$. By virtue of the precedent case, we get $\lambda \notin \sigma_{\pi}(\hat{A})$. In consequence, the operator $\lambda-\hat{A}$ is upper semi-Fredholm. Next, let $\pi: X \rightarrow X / M$ be the canonical projection. Using Lemma 2.2(ii), together with $\operatorname{dim}(N(\lambda-\hat{A}))<\infty$, it can be verified that

$$
N(\lambda-A) \subseteq \pi^{-1} N(\lambda-\hat{A}) \subseteq M+G \subseteq R^{\infty}(\lambda-A)+G
$$

for a finite-dimensional subspace $G$ of $X$. Now, we show that $R(\lambda-A)$ is closed. To do this, consider a sequence $\left(u_{n}\right)_{n}$ of elements of $R(\lambda-A)$, which converges to $u$. Then, there exists a sequence $\left(v_{n}\right)_{n}$ of elements of $D(A)$ such that 
$(\lambda-A) v_{n}=u_{n} \rightarrow u$. Since $R(\lambda-\hat{A})$ is closed, there exists $\widehat{w} \in D(\hat{A})$ such that $\widehat{u}=(\lambda-\hat{A}) \widehat{w}$. Hence,

$$
u-(\lambda-A) w \in R^{\infty}\left(e^{\lambda t_{0}}-T\left(t_{0}\right)\right) \subseteq R^{\infty}(\lambda-A) \subseteq R(\lambda-A) .
$$

Accordingly, $u \in R(\lambda-A)$. Consequently, the operator $\lambda-A$ is essentially semiregular. This proves the theorem.

The next theorem gives, under suitable assumptions, necessary and sufficient conditions for the generator of a strongly continuous semigroup to be semiregular. The proof can be found in [5].

THEOREM 2.3. Let $(T(t))_{t \geq 0}$ be a strongly continuous semigroup with generator $A$ and type $\omega_{0}$. If $(T(t))_{t \geq 0}$ satisfies any of the following conditions:

(a) $\lim _{t \rightarrow \infty}(1 / t)\|T(t)\|=0$;

(b) $\left|\omega_{0}\right|<\gamma(A)$,

then the following assertions are equivalent:

(i) $A$ is semiregular;

(ii) $0 \in \rho(A)$;

(iii) $H_{0}(A)=\{0\}$ and $R(A)$ is closed.

The following example shows that conditions (a) and (b) in Theorem 2.3 are needed for the conclusion.

EXAMPLE 2.4. Let $H$ be a Hilbert space with an orthonormal basis $\left\{e_{n}\right\}_{n=1}^{\infty}$. Let $A$ be the operator on $H$ defined by $A e_{n}=e_{n+1}, n=1,2, \ldots$, and let $T(t)=e^{t A}$ be the semigroup generated by $A$. It is well known (see, e.g., [16, Chapter 2, Theorems 4 and 6]) that $\sigma(A)=\{\lambda \in \mathbb{C},|\lambda| \leq 1\}$ and $\sigma_{\text {ap }}(A)=\{\lambda \in \mathbb{C},|\lambda|=1\}$. Thus, $A$ is semiregular but $0 \notin \rho(A)$.

We conclude this section by the following result.

THEOREM 2.5. Let $A$ be the generator of a strongly continuous semigroup $(T(t))_{t \geq 0}$ satisfying $\lim _{t \rightarrow \infty}(1 / t)\|T(t)\|=0$. The following assertions are equivalent:

(i) A is essentially semiregular;

(ii) A is upper semi-Fredholm.

Proof. (i) $\Rightarrow$ (ii). Since $A$ is essentially semiregular, there exists a finitedimensional subspace $G$ of $X$ such that $N(A) \subseteq R^{\infty}(A)+G$. As noticed in [13], we may assume that $G \subseteq N(A)$. Let $y \in N(A)$ and let $x \in D(A)$ and $g \in G$ such that $y=A x+g$. Using Lemma 2.2(i), we infer that

$$
T(t) x=x+\int_{0}^{t} T(s)(y-g) d s=x+t(y-g), \quad \forall t \geq 0 .
$$

Since $\lim _{t \rightarrow \infty}(1 / t)\|T(t)\|=0$, then $y=g$. In consequence, $N(A)=G$. This is the desired result.

(ii) $\Rightarrow$ (i). Obvious. 
REMARK 2.6. Theorems 2.3 and 2.5 follow under weaker assumptions (e.g., $\left.\lim _{t \rightarrow \infty} t^{-n}\|T(t)\|, n \in \mathbb{N}\right)$. This follows essentially from the fact that the spectrum of $A$ is contained in the left half-plane $\{\lambda \in \mathbb{C}, \operatorname{Re}(\lambda) \leq 0\}$.

3. Stability results. In this section, we give some stability results for strongly continuous semigroups. First, we introduce some relevant notations and terminologies. By $\mathbb{C}^{-}$we denote the open left half of the complex plane, that is, the set of all $\lambda \in \mathbb{C}$ such that $\operatorname{Re}(\lambda)<0$. A closed operator $A$ is called stable if $\sigma(A) \subseteq \mathbb{C}^{-}$. A strongly continuous semigroup $(T(t))_{t \geq 0}$ is said to be strongly stable if $\|T(t) x\| \rightarrow 0$ as $t \rightarrow \infty$ for all $x \in X$. We say that $(T(t))_{t \geq 0}$ is uniformly stable if $\|T(t)\| \rightarrow 0$ as $t \rightarrow \infty$. Recall that a strongly stable semigroup is necessarily bounded and has no pure imaginary point in the point spectrum of its generator. For a recent account of stability results of strongly continuous semigroups, we refer the reader to [6, Chapter V].

We begin with the following stability results.

THEOREM 3.1 [5]. Let $A$ be the generator of a bounded strongly continuous semigroup $(T(t))_{t \geq 0}$. If $\sigma_{\gamma}(A) \cap i \mathbb{R}=\varnothing$, then $(T(t))_{t \geq 0}$ is strongly stable.

THEOREM 3.2 [5]. Let A be the generator of a bounded strongly continuous semigroup $(T(t))_{t \geq 0}$. Then, the following assertions are equivalent:

(i) $(T(t))_{t \geq 0}$ is uniformly stable;

(ii) $\sigma_{\gamma}(T(t)) \cap \Gamma=\varnothing$,

where $\Gamma$ stands for the unit circle of $\mathbb{C}$.

In order to state the next result, we need to introduce the ultrapower semigroup $\widetilde{\mathscr{T}}$ of a given semigroup $\mathscr{T}=(T(t))_{t \geq 0}$.

Following [20, page 35] (see also [1,8]), we define the space $\ell_{0}^{\infty}(X)$ as the set of all bounded sequences $\left(x_{n}\right)_{n} \subseteq X$ such that

$$
\lim _{t \downarrow 0}\left(\sup _{n}\left\|T(t) x_{n}-x_{n}\right\|\right)=0
$$

The ultrapower semigroup $\tilde{\mathscr{T}}=(\tilde{T}(t))_{t \geq 0}$ is defined on the quotient space

$$
\tilde{X}:=\ell_{0}^{\infty}(X) / C_{0}(X)
$$

by

$$
\tilde{T}(t)\left(\left(x_{n}\right)_{n}+C_{0}(X)\right)=\left(T(t) x_{n}\right)_{n}+C_{0}(X),
$$

where $C_{0}(X)$ stands for the space of all sequences in $X$ that converge to 0 . 
The semigroup $\widetilde{\mathcal{T}}$ is, by construction, strongly continuous. Its generator $\tilde{A}$ is given by

$$
\begin{gathered}
D(\tilde{A})=\left\{\left(x_{n}\right)_{n}+C_{0}(X),\left(x_{n}\right)_{n} \in \ell_{0}^{\infty}(X), x_{n} \in D(A) \forall n,\left(A x_{n}\right)_{n} \in \ell_{0}^{\infty}(X)\right\}, \\
\tilde{A}\left(\left(x_{n}\right)_{n}+C_{0}(X)\right)=\left(A x_{n}\right)_{n}+C_{0}(X) .
\end{gathered}
$$

The spectra of $A$ and $\tilde{A}$ are related as follows:

$$
\sigma(\tilde{A})=\sigma(A), \quad \sigma_{\mathrm{p}}(\tilde{A})=\sigma_{\mathrm{ap}}(\tilde{A})=\sigma_{\mathrm{ap}}(A) .
$$

THEOREM 3.3. Let A be the generator of a bounded strongly continuous semigroup $\mathcal{T}=(T(t))_{t \geq 0}$. Then the following assertions are equivalent:

(i) $\sigma_{\gamma}(A) \cap i \mathbb{R}=\varnothing$;

(ii) $\sigma(A) \cap i \mathbb{R}=\varnothing$;

(iii) $A$ is stable;

(iv) for every $x^{\star} \in X^{\star}$ and for every $\beta \in \mathbb{R},\left\|R\left(\lambda+i \beta, A^{\star}\right) x^{\star}\right\|=$ $O(1)($ as $\lambda \rightarrow 0)$;

(v) $\tilde{\mathscr{T}}$ is strongly stable.

Proof. (i) $\Rightarrow$ (ii). It suffices to apply Theorem 2.3 to the rescaled semigroup $\left(e^{-i \lambda t} T(t)\right)_{t \geq 0}$ whose generator is $A-i \lambda$.

(ii) $\Rightarrow$ (i). Obvious.

(ii) $\Leftrightarrow$ (iii). This is an immediate consequence of the Hille-Yosida theorem [6, Chapter II, Theorem 3.8].

(ii) $\Leftrightarrow$ (iv). Applying [18, Theorem 3] to the rescaled semigroup $S(t)=$ $e^{-i \beta} T(t), \beta \in \mathbb{R}$, whose generator is $A-i \beta$, we can assert that condition (iv) is equivalent to $\sigma_{\mathrm{su}}\left(A^{\star}\right) \cap i \mathbb{R}=\varnothing$. This is equivalent to $\sigma_{\mathrm{ap}}(A) \cap i \mathbb{R}=\varnothing$. Using Theorem 2.3, we infer that $\sigma(A) \cap i \mathbb{R}=\varnothing$, which is the desired result.

(v) $\Rightarrow$ (ii). Since $\tilde{\mathscr{T}}$ is strongly stable, then $\sigma_{\mathrm{p}}(\tilde{A}) \cap i \mathbb{R}=\varnothing$. In consequence, $\sigma_{\text {ap }}(A) \cap i \mathbb{R}=\varnothing$. Arguing as above, we get $\sigma(A) \cap i \mathbb{R}=\varnothing$.

(ii) $\Rightarrow(v)$. This follows from Theorem 3.1 on the basis of (3.5).

REMARK 3.4. (1) It was shown in [3], under the hypothesis of Theorem 3.3, that the condition $\sigma(A) \subseteq \mathbb{C}^{-}$is equivalent to

$$
\sup _{t>0}\left\|\int_{0}^{t} e^{i \mu s} T(s) d s\right\|<\infty, \quad \forall \mu \in \mathbb{R}, \forall x \in X .
$$

(2) In the general case, the condition $\sigma(A) \cap i \mathbb{R}=\varnothing$ does not characterize, even in Hilbert spaces, the strong stability of the semigroup generated by $A$. The translation semigroup $T(t) f(x):=f(x+t), t \geq 0$, on $L^{2}\left(\mathbb{R}_{+}\right)$shows that this condition is not necessary for strong stability. In fact, this semigroup has the generator $A=d / d x$, and the spectrum of $A$ is the left half plane $\{\lambda \in \mathbb{C}: \operatorname{Re} \lambda \leq 0\}$, see [1, A.III, 2.4, page 66]. Hence, $\sigma(A) \cap i \mathbb{R}=i \mathbb{R}$ but 
$\lim _{t \rightarrow \infty}\|T(t) f\|=0$ for every $f \in L^{2}\left(\mathbb{R}_{+}\right)$. However, Theorem 3.3 shows that the condition $\sigma(A) \cap i \mathbb{R}=\varnothing$ characterizes completely the strong stability of the ultrapower extension of the semigroup generated by $A$.

COROLLARY 3.5. Let $A$ be the generator of a bounded strongly continuous semigroup $\mathcal{T}=(T(t))_{t \geq 0}$ on a reflexive Banach space $X$. Then, conditions (i), (ii), (iii), (iv), and ( $v$ ) of Theorem 3.3 are equivalent to

(vi) for every $x \in X$ and for every $\beta \in \mathbb{R},\|R(\lambda+i \beta, A) x\|=O(1)($ as $\lambda \rightarrow 0)$.

Proof. It is well known [19, Corollary 1.3.2] that the adjoint semigroup of a strongly continuous semigroup on a reflexive Banach space is again strongly continuous. It suffices to apply Theorem 3.3 to the adjoint semigroup whose generator is $A^{\star}$.

Note that, in reflexive Banach spaces, condition (vi) implies the strong stability of the bounded semigroup generated by $A$ (this follows from Corollary 3.5 and Theorem 3.1). In general Banach spaces setting, we have the following proposition.

Proposition 3.6. Let $A$ be the generator of a bounded strongly continuous semigroup $(T(t))_{t \geq 0}$, satisfying condition (vi). Then,

$$
\lim _{t \rightarrow \infty}\|T(t) x\|=0, \quad \forall x \in \overline{\bigcap_{\beta \in \mathbb{R}} R(i \beta-A)} .
$$

Proof. Condition (vi) implies that $\lim _{\lambda \rightarrow 0} \lambda R(\lambda, A-i \beta) x=0$ for all $x \in X$. By the abelian mean ergodic theorem [7, page 520], it follows that $R(i \beta-A)$ is dense in $X$ for all $\beta \in \mathbb{R}$. Hence,

$$
\bigcap_{\beta \in \mathbb{R}} R(i \beta-A)=\bigcap_{\beta \in \mathbb{R}}(i \beta-A)(\overline{R(i \beta-A)} \cap D(A)) .
$$

Using [2, Theorem 6.3(ii)] and the strong continuity of the semigroup, we get the desired result.

ACKNOWLEDGMENT. The authors would like to thank the referees for helpful suggestions and remarks.

\section{REFERENCES}

[1] W. Arendt, A. Grabosch, G. Greiner, U. Groh, H. P. Lotz, U. Moustakas, R. Nagel, F. Neubrander, and U. Schlotterbeck, One-Parameter Semigroups of Positive Operators, Lecture Notes in Mathematics, vol. 1184, Springer-Verlag, Berlin, 1986.

[2] C. J. K. Batty, R. Chill, and Y. Tomilov, Strong stability of bounded evolution families and semigroups, J. Funct. Anal. 193 (2002), no. 1, 116-139.

[3] C. Buşe and D. Barbu, Some remarks about the Perron condition for $C_{0}$ semigroups, An. Univ. Timişoara Ser. Mat.-Inform. 35 (1997), no. 1, 3-8. 
[4] Ph. Clément, H. J. A. M. Heijmans, S. Angenent, C. J. van Duijn, and B. de Pagter, One-Parameter Semigroups, CWI Monographs, vol. 5, North-Holland Publishing, Amsterdam, 1987.

[5] A. Elkoutri and M. A. Taoudi, Spectre singulier pour les générateurs des semigroupes [Singular spectrum for generators of semigroups], C. R. Acad. Sci. Paris Sér. I Math. 333 (2001), no. 7, 641-644 (French).

[6] K.-J. Engel and R. Nagel, One-Parameter Semigroups for Linear Evolution Equations, Graduate Texts in Mathematics, vol. 194, Springer-Verlag, New York, 2000.

[7] E. Hille and R. S. Phillips, Functional Analysis and Semi-Groups, American Mathematical Society Colloquium Publications, vol. 31, American Mathematical Society, Rhode Island, 1957.

[8] S. Z. Huang and F. Räbiger, Superstable $C_{0}$-semigroups on Banach spaces, Evolution Equations, Control Theory, and Biomathematics (Han sur Lesse, 1991) (Ph. Clément and G. Lumer, eds.), Lecture Notes in Pure and Appl. Math., vol. 155, Dekker, New York, 1994, pp. 291-299.

[9] T. Kato, Perturbation Theory for Linear Operators, Die Grundlehren der Mathematischen Wissenschaften, vol. 132, Springer-Verlag, New York, 1966.

[10] V. Kordula and V. Müller, The distance from the Apostol spectrum, Proc. Amer. Math. Soc. 124 (1996), no. 10, 3055-3061.

[11] M. Mbekhta, Résolvant généralisé et théorie spectrale [Generalized resolvents and spectral theory], J. Operator Theory 21 (1989), no. 1, 69-105 (French).

[12] _ On the generalized resolvent in Banach spaces, J. Math. Anal. Appl. 189 (1995), no. 2, 362-377.

[13] V. Müler, On the regular spectrum, J. Operator Theory 31 (1994), no. 2, 363-380.

[14] A. Ouahab and M. Mbekhta, Contribution à la théorie spectrale généralisée dans les espaces de Banach [A contribution to general spectral theory in Banach spaces], C. R. Acad. Sci. Paris Sér. I Math. 313 (1991), no. 12, 833-836 (French).

[15] A. Pazy, Semigroups of Linear Operators and Applications to Partial Differential Equations, Applied Mathematical Sciences, vol. 44, Springer-Verlag, New York, 1983.

[16] C. Pearcy (ed.), Topics in Operator Theory, Mathematical Surveys, vol. 13, American Mathematical Society, Rhode Island, 1979.

[17] V. Rakočevič, Generalized spectrum and commuting compact perturbations, Proc. Edinburgh Math. Soc. 36 (1993), no. 2, 197-209.

[18] S.-Y. Shaw, On the range of a closed operator, J. Operator Theory 22 (1989), no. 1, 157-163.

[19] J. van Neerven, The Adjoint of a Semigroup of Linear Operators, Lecture Notes in Mathematics, vol. 1529, Springer-Verlag, Berlin, 1992.

[20] _ The Asymptotic Behaviour of Semigroups of Linear Operators, Operator Theory: Advances and Applications, vol. 88, Birkhäuser Verlag, Basel, 1996.

Abdelkader Elkoutri: Department of Mathematics, Faculty of Sciences Semlalia, Cadi Ayyad University, BP 2390, Marrakesh 40000, Morocco

E-mail address: e1koutri@ucam. ac.ma

Mohamed Aziz Taoudi: Department of Mathematics, Faculty of Sciences Semlalia, Cadi Ayyad University, BP 2390, Marrakesh 40000, Morocco

E-mail address: taoudi@ucam.ac.ma 


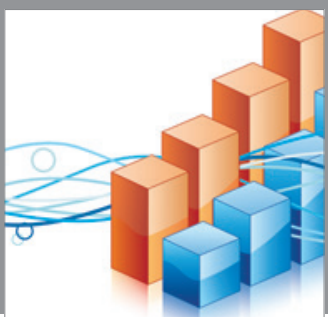

Advances in

Operations Research

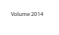

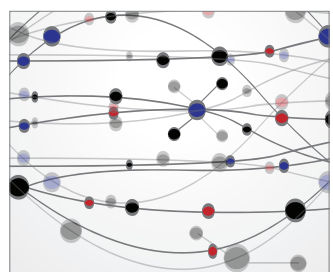

\section{The Scientific} World Journal
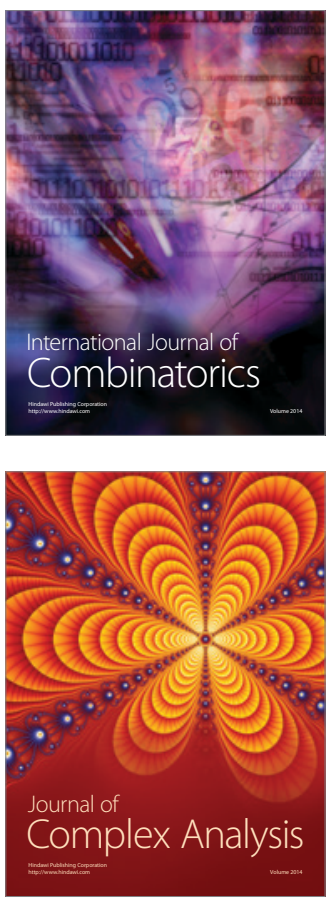

International Journal of

Mathematics and

Mathematical

Sciences
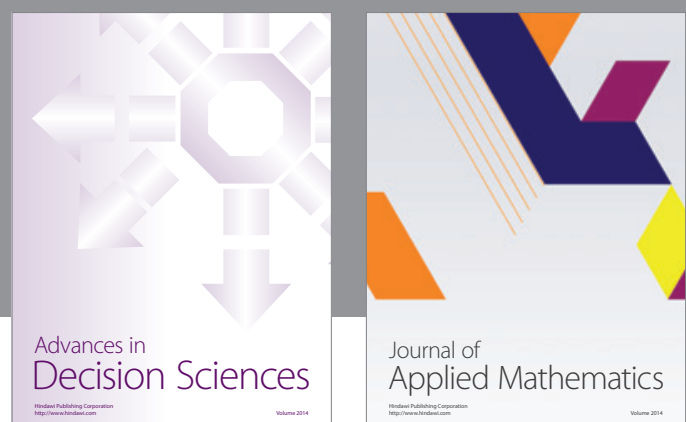

Journal of

Applied Mathematics
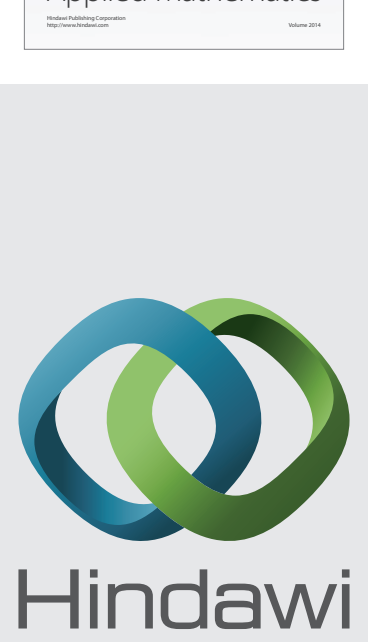

Submit your manuscripts at http://www.hindawi.com
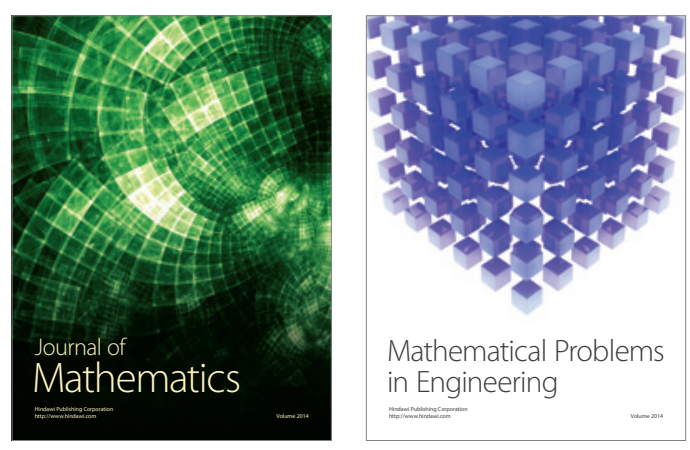

Mathematical Problems in Engineering
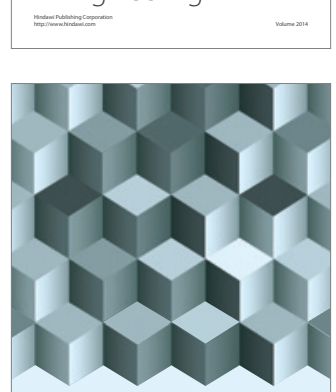

Journal of

Function Spaces
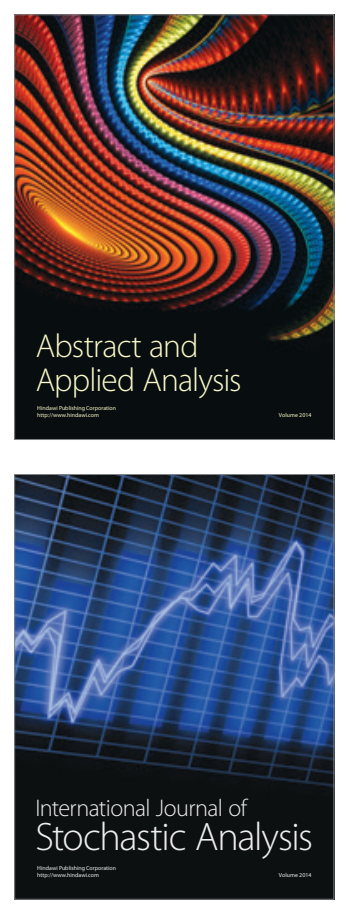

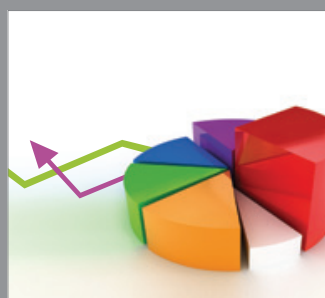

ournal of

Probability and Statistics

Promensencen
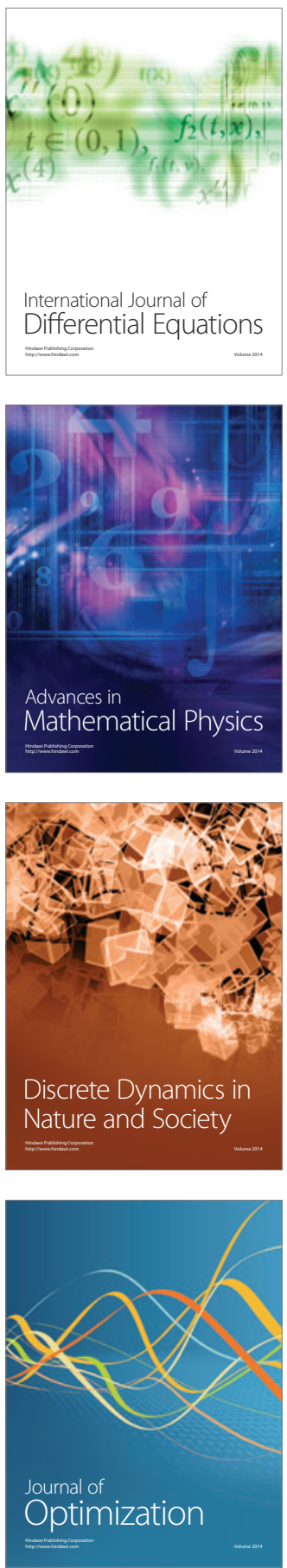